\title{
Negativity spectrum in 1D gapped phases of matter
}

\author{
Glen Bigan Mbeng, Vincenzo Alba, and Pasquale Calabrese \\ ${ }^{1}$ SISSA and INFN, via Bonomea 265, 34136 Trieste, Italy. \\ E-mail: gmbeng@sissa.it, valba@sissa.it and calabrese@sissa.it
}

\begin{abstract}
.
We investigate the spectrum of the partial transpose (negativity spectrum) of two adjacent regions in gapped one-dimensional models. We show that, in the limit of large regions, the negativity spectrum is entirely reconstructed from the entanglement spectrum of the bipartite system. We exploit this result in the XXZ spin chain, for which the entanglement spectrum is known by means of the corner transfer matrix. We find that the negativity spectrum levels are equally spaced, the spacing being half that in the entanglement spectrum. Moreover, the degeneracy of the spectrum is described by elegant combinatorial formulas, which are related to the counting of integer partitions. We also derive the asymptotic distribution of the negativity spectrum. We provide exact results for the logarithmic negativity and for the moments of the partial transpose. They exhibit unusual scaling corrections in the limit $\Delta \rightarrow 1^{+}$ with a corrections exponent which is the same as that for the Rényi entropies.
\end{abstract}




\section{Introduction}

In recent years entanglement witnesses became invaluable diagnostic tools to understand complex behaviours in quantum many-body systems [1-4]. For a bipartite system in a pure state, the von Neumann entanglement entropy and the Rényi entropies are appropriate entanglement measures, but this is not the case for a system in a mixed state. For instance, in finite temperature systems the entanglement entropy cannot distinguish the quantum correlations (entanglement) from the classical ones due to thermal fluctuations. A similar issue arises when quantifying the mutual entanglement between two non-complementary regions (see Figure 1). As the two intervals are in a mixed state, the von Neumann entropy and the mutual information fail to quantify their mutual entanglement. Physically, the mutual information cannot distinguish the entanglement between the two intervals from the correlations induced by the interaction with the environment.

Although several entanglement measures for mixed states exist, we will focus on the logarithmic negativity [5-10] because of its universal features. This is defined as follows. Let us consider a system in a pure state $|\psi\rangle$. Given a tripartition of the system as $A_{1} \cup A_{2} \cup B$, with $A \equiv A_{1} \cup A_{2}$ the region of interest (as in Figure 1 in one dimension), the reduced density matrix $\rho_{A}$ is defined as $\rho_{A} \equiv \operatorname{Tr}_{B} \rho$, with $\rho=|\psi\rangle\langle\psi|$. The logarithmic negativity $\mathcal{E}_{A_{1}: A_{2}}$ is

$$
\mathcal{E}_{A_{1}: A_{2}} \equiv \ln || \rho_{A_{1} \cup A_{2}}^{T_{2}} \|_{1}=\ln \operatorname{Tr}\left|\rho_{A_{1} \cup A_{2}}^{T_{2}}\right| .
$$

Here $\rho_{A_{1} \cup A_{2}}^{T_{2}}$ is the partially transposed reduced density matrix (partial transpose) with respect to $A_{2}$. This is defined from $\rho_{A}$ as $\left\langle\varphi_{1} \varphi_{2}\left|\rho_{A}^{T_{2}}\right| \varphi_{1}^{\prime} \varphi_{2}^{\prime}\right\rangle \equiv\left\langle\varphi_{1} \varphi_{2}^{\prime}\left|\rho_{A}\right| \varphi_{1}^{\prime} \varphi_{2}\right\rangle$, with $\left\{\varphi_{1}\right\}$ and $\left\{\varphi_{2}\right\}$ two bases for $A_{1}$ and $A_{2}$, respectively. The symbol $\|\cdot\|_{1}$ in (1) denotes the trace norm. In contrast with the reduced density matrix, which is positive semidefinite,

$\rho_{A}^{T_{2}}$ has both positive and negative eigenvalues. Given the normalisation $\operatorname{Tr} \rho_{A_{1} \cup A_{2}}^{T_{2}}=1$, $\mathcal{E}_{A_{1}: A_{2}}$ can be rewritten as a sum over the negative eigenvalues of the partial transpose, a property from which the name negativity comes from.

The logarithmic negativity has been characterised analytically for a generic $1+1$ dimensional Conformal Field Theory (CFT) [11-13]. For two or more disconnected intervals it depends on the full operator content of the CFT [11, 12], similarly to the entanglement entropy [14]. A remarkable feature is that the negativity is scale invariant at quantum critical points $[11,12,15-17]$. Its properties are also known at finite temperature [18], in CFTs with large central charge [19], disordered spin chains [20], out-of-equilibrium models [21-24], holographic [25] and massive quantum field theories [26, 27], topologically ordered phases [28, 29], Kondo-like systems [30-32], and Chern-Simons theories [33,34]. The negativity can be calculated analytically for free-bosonic models [35], also in $d>1$ dimensions [36, 37]. However, no results are available for free fermions, despite recent progress [38-45]. The negativity can be obtained numerically in tensor network simulations [13,15,20]. Finally, the full spectrum of the partial transpose has been studied recently for systems described by a CFT [46] (see Ref. 47 for a proposal to measure the negativity spectrum with quantum circuits). 


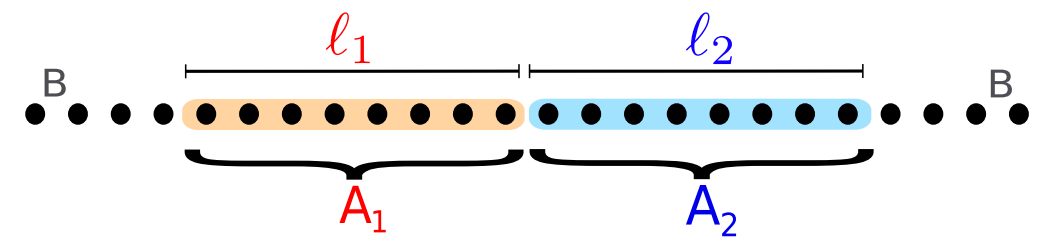

Figure 1. Tripartition of a one-dimensional chain considered in this work. The chain is divided into two adjacent non-complementary intervals $A \equiv A_{1} \cup A_{2}$. The rest of the chain is denoted as $B$. Here $\ell_{1}$ and $\ell_{2}$ are the lengths of the two intervals. We always consider the situation with $\ell_{1}=\ell_{2}=\ell$.

The distribution of the positive and negative eigenvalues of the partial transpose has been characterised analytically and, similar to the eigenvalues of the reduced density matrix [48] (entanglement spectrum), it has been shown that the negativity spectrum is universal and depends only on the central charge of the CFT.

Here we investigate the negativity spectrum in the ground state of one-dimensional gapped phases of matter. For the entanglement spectrum in gapped phases, a detailed analysis has been already presented $[49,50]$. We focus on the tripartition with two adjacent intervals embedded in a spin chain (as in Figure 1). Our main result is that for generic gapped systems, in the limit of large intervals, the negativity spectrum can be constructed entirely from the entanglement spectrum of the bipartition of the system in two semi-infinite halves. This reflects two important physical facts. First, the negativity spectrum is determined by degrees of freedom living at the boundaries between the subsystems, similarly to what happens for the entanglement spectrum [50]. Second, due to the finite bulk correlation length, near the boundary between $A_{1}$ and $A_{2}$ the subsystem $A_{1} \cup A_{2}$ can be effectively described by a pure state.

We exploit these ideas to derive the negativity spectrum of the XXZ spin chain from its entanglement spectrum, which is known exactly [49-54] from Corner Transfer Matrix (CTM) techniques. The anisotropic Heisenberg spin chain (XXZ chain) is defined by the Hamiltonian

$$
H_{X X Z}=\sum_{i=1}^{L}\left[S_{i}^{x} S_{i+1}^{x}+S_{i}^{y} S_{i+1}^{y}+\Delta S_{i}^{z} S_{i+1}^{z}\right],
$$

where $S_{i}^{\alpha} \equiv \sigma_{i}^{\alpha} / 2$, with $\sigma_{i}^{\alpha}$ the Pauli matrices, are spin- $\frac{1}{2}$ operators acting on the site $i$ of the chain, and $\Delta$ the anisotropy parameter. For $-1<\Delta \leq 1$ the spectrum of the XXZ chain is gapless, and its low-energy part is described by a $c=1$ CFT. For $\Delta>1$, which is the regime of interest here, the model is in the gapped antiferromagnetic regime.

The manuscript is organised as follows. In section 2 we show the construction of the partial transpose in the ground state of generic gapped systems. Section 3 describes how the negativity spectrum is constructed starting from the moments of the partial transpose and the entanglement spectrum of a bipartition. In section 4 we use these results to derive the structure of the negativity spectrum of the gapped XXZ chain. In section 5 we present some analytic results for the logarithmic negativity and the 
(a)
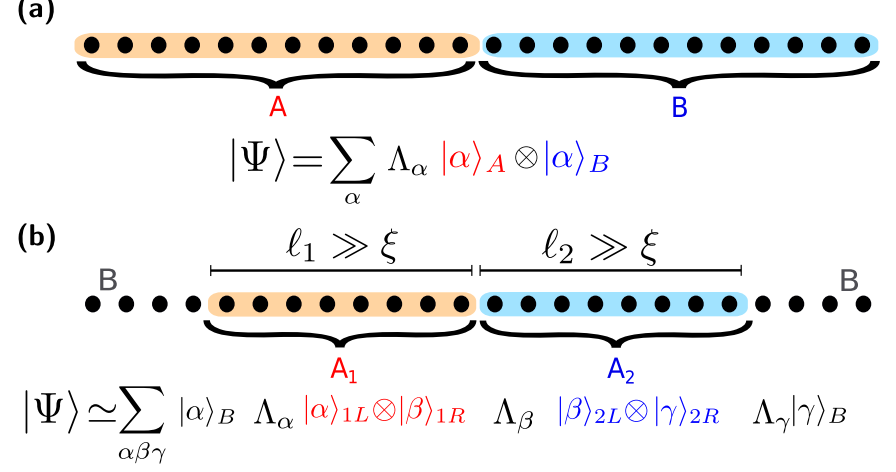

(c)

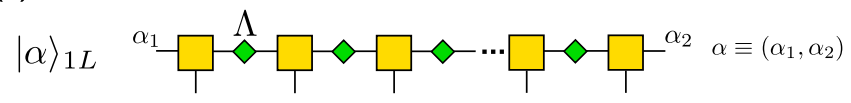

(d)

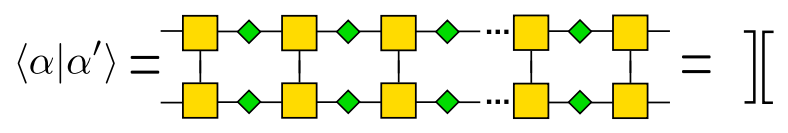

Figure 2. Logarithmic negativity of two adjacent intervals $A_{1}$ and $A_{2}$. (a) A bipartition of the chain as $A \cup B$ with the Schmidt decomposition of the groundstate wavefunction. $|\alpha\rangle_{A(B)}$ are two orthonormal bases for part $A(B), \Lambda_{\alpha}$ are the Schmidt values. (b) The tripartition used in this work. The length of the two intervals $\ell_{1}\left(\ell_{2}\right)$ is much larger than the correlation length $\xi$. In this regime, the entanglement is "localised" close to the three edges and an approximate decomposition for this tripartition is reported below the cartoon. Here the $\Lambda$ s are the Schmidt coefficients for the three bipartitions in semi-infinite chains at each edge (alike in (a)), while $|\alpha\rangle_{1 L}$ and $|\beta\rangle_{1 R}$ are the two Schmidt bases for the Hilbert space around the left and right boundary of $A_{1}$ (and similarly the others kets). (c) Matrix Product State representation for $|\alpha\rangle_{1 L}$. The squares denote the matrices $T$ (cf. (3)), rhombi the matrix $\Lambda$. (d) Orthogonality of the basis $|\alpha\rangle_{1 L}$. For $\ell_{1} \gg \xi$, one has $\left\langle\alpha \mid \alpha^{\prime}\right\rangle=\delta_{\alpha \alpha^{\prime}}$.

moments of the partial transpose. In section 6 we analyse the scaling of the negativity and of the moments of the partial transpose in the limit $\Delta \rightarrow 1^{+}$, and investigate the presence of unusual corrections to the scaling. In section 7 we derive analytic results for the distribution of the negativity spectrum in the XXZ chain. Our conclusions are in section 8 .

\section{Partially transposed reduced density matrix in gapped phases}

In this section we show that in the limit of large subsystems the negativity spectrum of two adjacent intervals can be reconstructed entirely from the entanglement spectrum of the bipartition of the system in two semi-infinite halves. The main physical idea is very simple and a very similar one was used for the negativity in [26]. Since we are interested in a tripartition in the regime in which both the finite intervals $\ell_{1,2}$ are much larger than the finite correlation length $\xi$, the entanglement is localised around the three edges of the tripartition, i.e. at the boundary between $B$ and $A_{1}$, that between $A_{1}$ and $A_{2}$, and 


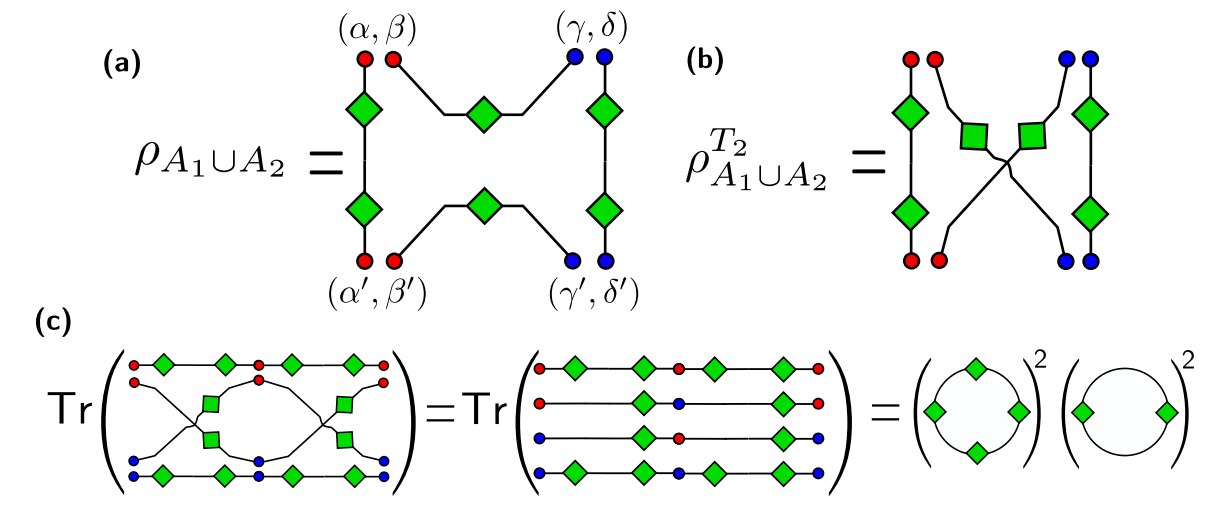

Figure 3. Matrix Product State representation for the reduced density matrix $\rho_{A_{1} \cup A_{2}}$ and the partial transpose $\rho_{A_{1} \cup A_{2}}^{T_{2}}$. The rhombi are the diagonal matrices containing the singular values (entanglement spectrum). Note in (b) that the effect of the partial transposition is to swap $\gamma \leftrightarrow \gamma^{\prime}$. The partial transposition is ineffective on $\delta \delta^{\prime}$. (c) The second moment of the partial transpose: pictorial proof of the identity $\operatorname{Tr}\left(\rho_{A_{1} \cup A_{2}}^{T_{2}}\right)^{2}=\left(\operatorname{Tr} \rho_{A_{1}}^{2}\right)^{2} \operatorname{Tr}\left(\rho_{A_{1}}\right)^{2}$. Note the untwisting of the tensor lines in the intermediate step.

between $A_{2}$ and $B$ (see Fig. 2). Consequently, the state is well approximated (with exponential accuracy in $\ell_{i} / \xi$ ) by a triple sum on the three bipartitions (which involves the same Schmidt coefficients) and the negativity spectrum is simply written in terms of the entanglement spectrum. Having explained the main idea, in the following we make the argument more rigorous employing the language of Matrix Product States (MPS). We restrict ourselves to spin- $\frac{1}{2}$ models (spin chains) with open boundary conditions, but our arguments are straightforwardly generalised to other situations.

In the MPS framework the ground state of a generic spin chain is represented as

$$
|\Psi\rangle=\sum_{\sigma_{1}, \ldots, \sigma_{L}} T_{\nu_{1}}^{\sigma_{1}} T_{\nu_{1}, \nu_{2}}^{\sigma_{2}} \cdots T_{\nu_{L}}^{\sigma_{L}}\left|\sigma_{1}, \ldots, \sigma_{L}\right\rangle
$$

Here $\sigma_{i}= \pm 1$ are the physical indices, which represent the states in the local Hilbert space, while $1 \leq \nu_{i} \leq \chi_{i}$ are the virtual indices, with $\chi_{i}$ the local bond dimension of the MPS. In (3), $T_{\nu_{1}}^{\sigma_{1}}$ and $T_{\nu_{L}}^{\sigma_{L}}$ are vectors, whereas for $1<i<L, T_{\nu_{i}, \nu_{i+1}}^{\sigma_{i}}$ are $\chi_{i} \times \chi_{i}$ matrices. The sum over repeated indices is assumed. Given a bipartition of the chain as $A \cup B$ (see Figure $2(\mathrm{a})$ ), the Schmidt decomposition allows us to rewrite $|\Psi\rangle$ as

$$
|\Psi\rangle=\sum_{\alpha} \Lambda_{\alpha}|\alpha\rangle_{A} \otimes|\alpha\rangle_{B}
$$

Here $|\alpha\rangle_{A(B)}$ are orthonormal bases (Schmidt vectors) for parts $A$ and $B$ and $\Lambda_{\alpha}$ is the diagonal matrix with the Schmidt values. The non-zero eigenvalues of the reduced density matrix $\rho_{A}$ are given as $\Lambda_{\alpha}^{2}$. For translational invariant systems (for instance for the half-infinite chain), the tensors $T_{\nu_{i}, \nu_{i+1}}^{\sigma_{i}}$ do not depend on the site $i$.

Let us now consider the tripartition of the chain $A_{1} \cup A_{2} \cup B$ as in Figure 2 (b), i.e., two adjacent intervals and the rest, with the lengths of the two intervals being much larger than the bulk correlation length $\xi$. The idea of the approach is to construct an 
approximate basis for the tripartite system. For gapped systems the eigenvectors of the reduced density matrix and of its partial transpose are expected to be localised at the boundary between the two subsystems and between the subsystems and the rest. This suggests that a useful orthonormal basis for $A_{1}$ can be constructed as $|\alpha\rangle_{1 L} \otimes|\beta\rangle_{1 R}$, with $|\alpha\rangle_{1 L}$ and $|\beta\rangle_{1 R}$ the Schmidt vectors appearing in (4) (see also Figure 2). The tensor product structure of the basis reflects the factorisation of the two edges of $A_{1}$, which relies on the condition $\ell_{1} \gg \xi$. Similarly, for $A_{2}$ one can construct the orthonormal basis as $|\beta\rangle_{2 L} \otimes|\gamma\rangle_{2 R}$, where, again, $|\beta\rangle_{2 L}$ and $|\gamma\rangle_{2 R}$ are the same Schmidt vectors in (4). This allows one to decompose the wavefunction of the system as

$$
|\Psi\rangle \simeq \sum_{\alpha, \beta, \gamma}|\alpha\rangle_{B} \Lambda_{\alpha}|\alpha\rangle_{1 L} \otimes|\beta\rangle_{1 R} \Lambda_{\beta}|\beta\rangle_{2 L} \otimes|\gamma\rangle_{2 R} \Lambda_{\gamma}|\gamma\rangle_{B}
$$

where $|\alpha\rangle_{B}$ and $|\gamma\rangle_{B}$ are elements of the Schmidt basis for part $B$ of the chain and $\Lambda_{\alpha}$ is the Schmidt coefficient of the bipartition (4). Eq. (5), in the regime $\ell_{1}, \ell_{2} \gg \xi$, has an exponential accuracy, i.e. the error is as small as $e^{-\ell_{i} / \xi}$, as argued for the negativity in Ref. 26.

The decomposition (5) of $|\Psi\rangle$ is illustrated pictorially in Figure 2 (c), using the MPS formalism to represent the vectors $|\alpha\rangle_{1 L}$. Similar representations hold for the other vectors appearing in (5). The square boxes in Figure 2 (c) represent the tensors $T_{\nu_{i}, \nu_{i+1}}^{\sigma_{i+1}}$ (cf. (3)), the rhombi the diagonal matrix $\Lambda_{\alpha}$ as in (4). The vertical dangling bonds are the physical indices $\sigma_{i}$ and are summed over. The index $\alpha$ is a composite index $\alpha \equiv\left(\alpha_{1}, \alpha_{2}\right)$, with $\alpha_{1}, \alpha_{2}$ the virtual indices at the edges of the MPS. The length of the MPS (number of tensor in Figure 2 (c)) has to be much larger than the correlation length. Note that since $|\alpha\rangle_{1 L}$ are constructed from the Schmidt vectors for the bipartition, they are (approximately) orthonormal. The orthonormality condition $\left\langle\alpha \mid \alpha^{\prime}\right\rangle=\delta_{\alpha, \alpha^{\prime}}$ is illustrated in Figure 2 (d).

Using (5), it is straightforward to derive the MPS representation of the reduced density matrix $\rho_{A_{1} \cup A_{2}}$. This is reported in Figure $3(\mathrm{a})$. The composite indices $(\alpha, \beta)$ and $\left(\alpha^{\prime}, \beta^{\prime}\right)$ refer to $A_{1}$, whereas $(\gamma, \delta)\left(\gamma^{\prime}, \delta^{\prime}\right)$ are for $A_{2}$. Specifically, the indices $\beta, \gamma$ refer to the degrees of freedom living at the interface between $A_{1}$ and $A_{2}$, while $\alpha, \delta$ to the ones lying at the interface between $A_{1} \cup A_{2}$ and the rest of the system. Again, the rhombi denote the Schmidt values $\Lambda_{\alpha}$ for the bipartite system (Figure 2 (a)). Clearly, $\rho_{A_{1} \cup A_{2}}$ is a tensor product of three terms, corresponding to the three different boundaries present in the system. At this point the partial transpose with respect to $A_{2}$ is trivially derived since it acts as a global transposition on the indices $\alpha, \alpha^{\prime}$ and $\delta, \delta^{\prime}$. The only non trivial effect is the swap $\gamma \leftrightarrow \gamma^{\prime}$. The structure of $\rho_{A_{1} \cup A_{2}}^{T_{2}}$ is illustrated pictorially in Figure 3 (b).

\section{Negativity spectrum from the moments of the partial transpose}

In the above section we showed that the partial transpose of two adjacent intervals (in a tripartite system) can be written in terms of the reduced density matrix of a semiinfinite half of the gapped system. Thus a similar relation should holds also between 
the entire negativity spectrum of the tripartition and the entanglement spectrum of the bipartition. This relation is obtained in an easy way exploiting the moments $\operatorname{Tr}\left(\rho_{A_{1} \cup A_{2}}^{T_{2}}\right)^{n}$ that can be also written in terms of the moments of the reduced density matrix for the bipartition, as in Figure 2 (a). By very simple algebra, it is straightforward to derive the following identities depending on the parity of $n$ :

$$
\operatorname{Tr}\left(\rho_{A_{1} \cup A_{2}}^{T_{2}}\right)^{n}=\left(\operatorname{Tr} \rho_{A_{1}}^{n}\right)^{2} \times\left\{\begin{array}{ccc}
\left(\operatorname{Tr} \rho_{A_{1}}^{n_{e} / 2}\right)^{2} & n_{e} & \text { even }, \\
\operatorname{Tr} \rho_{A_{1}}^{n_{o}} & n_{o} & \text { odd },
\end{array}\right.
$$

where $n_{e}$ and $n_{o}$ are even and odd integers, respectively. A pictorial derivation of (6) for $n=2$ is reported in Figure 3 (c). The contributions of the straight horizontal lines is $\left(\operatorname{Tr}\left(\rho_{A_{1}}\right)^{2}\right)^{2}$ (top and bottom lines in the left member in Figure 3 (c)). This is clearly generalised as $\left(\operatorname{Tr} \rho_{A_{1}}^{n}\right)^{2}$ for arbitrary $n$, and it does not depend on the parity of $n$. The non-trivial contribution to the moments originates from the "entangled" lines in Figure 3 (c). For $n=2$, in taking the trace the two lines can be "disentangled" into two independent circles (second term in the rightmost member in the Figure). For generic even $n$ one obtains two independent closed diagrams ("circles"), each containing $n$ rhombi. This corresponds to $\left(\operatorname{Tr} \rho_{A_{1}}^{n / 2}\right)^{2}$. On the other hand, for $n$ odd the "entangled" diagrams are topologically equivalent to a single closed circle with $2 n$ rhombi, which is $\operatorname{Tr} \rho_{A_{1}}^{n}$. The equations in (6) are similar to the relations valid for the moments of the partial transpose for a bipartite pure state $[11,12]$

$$
\operatorname{Tr}\left(\rho_{A_{1} \cup A_{2}}^{T_{2}}\right)^{n}= \begin{cases}\left(\operatorname{Tr} \rho_{A_{1}}^{n_{e} / 2}\right)^{2} & n_{e} \text { even }, \\ \operatorname{Tr} \rho_{A_{1}}^{n_{o}} & n_{o} \text { odd } .\end{cases}
$$

Clearly, the relations (7) are the same as (6) apart for a multiplicative factor. This is physically expected because for $\ell_{1}, \ell_{2} \gg \xi$ the system around the boundary between $A_{1}$ and $A_{2}$ is effectively in a pure state. Moreover, Eq. (6) shows that $\operatorname{Tr}\left(\rho_{A_{1} \cup A_{2}}^{T_{2}}\right)^{n}$ depends on the degrees of freedom at the boundary between $A$ and $B$ through the term $\left(\operatorname{Tr} \rho_{A_{1}}^{n}\right)^{2}$. This reflects that for $n \neq 1$ the moments of the partial transpose are not good measures of the mutual entanglement between $A_{1}$ and $A_{2}$. Note, however, that the logarithmic negativity, which is obtained from (6) by taking the limit $n_{e} \rightarrow 1$, depends only on the degrees of freedom at the boundary between $A_{1}$ and $A_{2}$, as it should.

Finally, the relations in (6) imply that the negativity spectrum can be completely derived from the entanglement spectrum of a bipartite system, since they represent an infinite set of equations for the eigenvalues of the partial transpose. Denoting as $\mu_{i}$ the eigenvalues of the reduced density matrix $\rho_{A_{1}}$ of the half-infinite system, the eigenvalues of the partial transpose $\lambda_{i, j, k, l}$ can be written as

$$
\lambda_{i, j, k, l}=\mu_{i} \mu_{j} \begin{cases}\sqrt{\mu_{k} \mu_{l}} & k<l, \\ \mu_{k} & k=l, \\ -\sqrt{\mu_{k} \mu_{l}} & k>l .\end{cases}
$$

This can be proved by verifying that the $\lambda_{i, j, k, l}$ defined in (8) satisfy (6). 


\section{Structure of the negativity spectrum in the gapped Heisenberg chain}

The goal of this section is to apply (8) to derive the negativity spectrum of the gapped antiferromagnetic XXZ chain. To this purpose, the key ingredient is the half-chain entanglement spectrum, which can be constructed completely using the Corner Transfer Matrix (CTM) techniques [55].

\subsection{Entanglement spectrum of the XXZ chain via the Corner Transfer Matrix}

In this section we review the construction of the entanglement spectrum using the CTM approach [52-54]. Given a two dimensional statistical physics model, the CTM $\hat{M}$ is defined as the transfer matrix connecting a horizontal row to a vertical one. Specifically, the matrix elements of $\hat{M}$ are the partition functions on the lower left quadrant with fixed boundary conditions on the $x$ and $y$ axis. The reduced density matrix $\rho_{A}$ of the half-infinite system is related to $\hat{M}$ as

$$
\rho_{A}=\frac{\hat{M}^{4}}{\operatorname{Tr} \hat{M}^{4}} .
$$

For the gapped XXZ chain, in the infinite lattice limit, the reduced density matrix can be rewritten as

$$
\rho_{A}=\frac{e^{-H_{C T M}}}{\operatorname{Tr} e^{-H_{C T M}}},
$$

where $H_{C T M}$ is a modular (or entanglement) Hamiltonian. $H_{C T M}$ can be recast in the free-fermion form

$$
H_{C T M}=\sum_{j=0}^{\infty} \epsilon_{j} \hat{n}_{j},
$$

with $\hat{n}_{j}$ a fermionic number operator with eigenvalues 0 and 1 , and $\epsilon_{j}$ the "singleparticle" entanglement spectrum levels [52]

$$
\epsilon_{j}=2 j \epsilon, \quad j \in \mathbb{N}, \quad \text { and } \epsilon \equiv \operatorname{arccosh} \Delta .
$$

The entanglement spectrum is obtained by filling in all the possible ways the single particle levels $\epsilon_{j}$ in (11) (i.e., setting all $\hat{n}_{j}$ equal either to 0 or 1 ). The resulting levels are equally spaced with spacing $2 \epsilon$. It is also clear from (11) and (12) that these levels are highly degenerate. The degeneracy of the level $\zeta=2 \epsilon n$ with $n=\sum_{j} j$ (cf. (12)) is obtained by counting the number of ways of obtaining $n$ as a sum of smaller nonrepeated integers (including zero). This is the problem of counting the number $q(n)$ of (restricted) partitions of $n$. The number $q(n)$ is conveniently generated as a function of $n$ via the generating function

$$
G(z) \equiv \sum_{k=0}^{\infty} q(k) z^{k}=\prod_{k=1}^{\infty}\left(1+z^{k}\right) .
$$

Thus, $q(n)$ is obtained from (13) as the coefficient of the monomial $z^{n}$. The ES degeneracy is given by $2 q(n)$, with the factor two coming from the overall double 

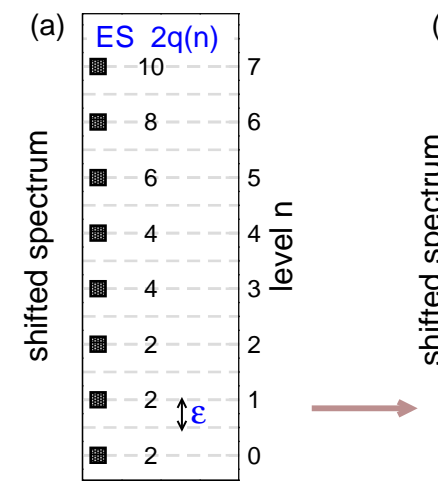

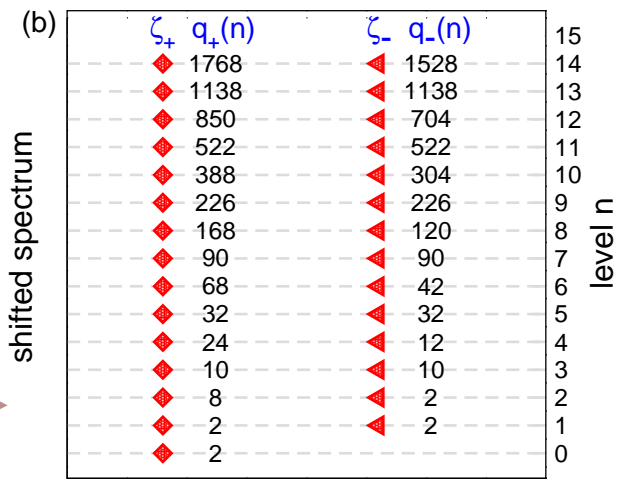

Figure 4. Survey of theoretical results for the negativity spectrum of two adjacent intervals in the gapped XXZ spin chain. The cartoon shows the levels $\zeta_{ \pm}$in the positive and negative sectors of the spectrum (rhombi and triangles, respectively). The entanglement spectrum levels for two half-infinite lines are reported for comparison (squares). All the spectra are shifted by the lowest level. The numbers accompanying the symbols denote the degeneracy. For the ES this is $2 q(n)$, with $q(n)$ the number of distinct integer partitions of $n . q_{ \pm}$denotes the degeneracy of $\zeta_{ \pm}$, with $q_{+}(n)=q_{-}(n)$ for odd $n$. Both for the negativity and the entanglement spectrum, the levels are equally spaced, the spacing being $\epsilon \equiv \operatorname{arccosh} \Delta$ and $2 \epsilon$, respectively.

degeneracy given by the zero mode $n_{0}=0,1$ with $\epsilon_{0}=0 . \ddagger$ Clearly, the ES degeneracy is given by $2 q(n)$ for those bipartitions which have one boundary between $A$ and $B$ (two half-infinite lines or finite ones with lengths $\ell_{i} \gg \xi$ ). For bipartitions with two boundaries (e.g. an interval embedded in an infinite or a finite system) the degeneracies are more complicated [50].

We mention that the corner transfer matrix techniques have been applied to the study of entanglement entropy and spectrum also to other exactly solvable models [56-60].

\subsection{Negativity spectrum}

Here we derive the structure of the negativity spectrum in the gapped Heisenberg spin chain for two adjacent finite intervals of lengths much larger than the correlation length. We denote by $\zeta_{+}=-\ln \lambda_{+}$all the levels corresponding to the positive eigenvalues $\lambda_{+}$ of the partial transpose and by $\zeta_{-}=-\ln \left|\lambda_{-}\right|$those corresponding to the negative eigenvalues $\lambda_{-}$. Eq. (8) implies that both $\zeta_{+}$and $\zeta_{-}$are equally spaced, the spacing being $\epsilon$ (cf. (12)), while for the entanglement spectrum the spacing is $2 \epsilon$. This difference is due to the square root in (8).

The structure of the negativity spectrum is presented in Figure 4. The Figure shows on

$\ddagger$ Physically this is due to the fact that in our construction the CTM selects the (equally probable) linear combination of the two degenerate ground states of the XXZ spin chain for $\Delta>1$ (i.e. Neel plus AntiNeel at $\Delta=\infty)$. The degeneracy of the ES levels for symmetry breaking states is just $q(n)$ as in Ref. 50. In the CTM approach, the symmetry breaking states can be obtained from (11) by letting the sum starting for $j=1$ instead of $j=0$, which correspond to fixing a boundary condition at the origin [55]. 
the left the entanglement spectrum levels (square symbols), shifted by the lowest level. The accompanying numbers are the level degeneracies, which are given as $2 q(n)$, with $q(n)$ the number of restricted integer partitions of $n$.

The rhombi and the triangles show the negativity spectrum levels corresponding to positive and negative eigenvalues of $\rho_{A_{1} \cup A_{2}}^{T_{2}}$. The corresponding degeneracies $q_{ \pm}(n)$ are also reported. Both $\zeta_{+}$and $\zeta_{-}$are shifted by the lowest level. The degeneracy sequences $q_{ \pm}(n)$ are constructed from (8), and they are conveniently derived from the generating functions

$$
G_{ \pm}(x) \equiv \sum_{k=0}^{\infty} q_{ \pm}(k) x^{k}=G^{2}\left(x^{2}\right)\left[G^{2}(x) \pm G\left(x^{2}\right)\right] .
$$

Here $G(x)$ is the same generating function as in (13). The terms $G^{2}\left(x^{2}\right)$ and $G^{2}(x)$ in (14) correspond to $\mu_{i} \mu_{j}$ and $\sqrt{\mu_{k} \mu_{l}}$ in (8), respectively. The second term in the square bracket in (14) properly counts the diagonal term with $k=l$ in (8). A nontrivial consequence of (14) is that $q_{+}(n)=q_{-}(n)$ for $n$ odd, as obvious from Figure 4. Thus the negativity spectrum levels can be written as

$$
\zeta=\zeta_{0}+n \epsilon, \quad n=0,1, \ldots,
$$

with $\zeta_{0}$ the lowest levels which is obtained by imposing the normalisation $\operatorname{Tr} \rho_{A_{1} \cup A_{2}}^{T_{2}}=1$. Indeed we have

$$
\operatorname{Tr} \rho_{A_{1} \cup A_{2}}^{T_{2}}=\sum \lambda_{+}+\sum \lambda_{-}=e^{-\zeta_{0}} \sum_{k=0}^{\infty}\left[q_{+}(k)-q_{-}(k)\right] e^{-k \epsilon}=1,
$$

which implies

$\zeta_{0}=\ln \sum_{k=0}^{\infty}\left[q_{+}(k)-q_{-}(k)\right] e^{-k \epsilon}=\ln \left[G_{+}\left(e^{-\epsilon}\right)-G_{-}\left(e^{-\epsilon}\right)\right]=3 \ln \left(G\left(e^{-2 \epsilon}\right)\right)+\ln 2$,

with $G_{ \pm}(z)$ defined in (14). It follows then that the largest eigenvalue of $\operatorname{Tr} \rho_{A_{1} \cup A_{2}}^{T_{2}}$ is

$$
\lambda_{0}=\frac{1}{2\left(G\left(e^{-2 \epsilon}\right)\right)^{3}} \text {. }
$$

\subsection{Numerical checks using TEBD}

We now provide some numerical checks of the results presented for both the values and the degeneracy of the negativity spectrum level summarised in Figure 4. Figure 5 shows time evolving block decimation [61,62] (TEBD) data for the negativity spectrum of the XXZ chain at $\Delta=2$. The data are for a chain with $L=128$ sites and open boundary conditions. The two blocks $A_{1}$ and $A_{2}$ are both composed of $L / 4$ spins. The simulations in Figure 5 (a) and (b) are performed at fixed bond dimension $\chi=70$. The ground state of the $X X Z$ chain is obtained by imaginary time evolution of an initial MPS via the Trotter decomposition of, as usual in TEBD. For a generic tripartition, the partial transpose and the negativity spectrum are constructed using the method described in Ref. 20, which relies on the MPS representation of the ground state of the XXZ chain. The calculation of the negativity spectrum involves the diagonalisation 

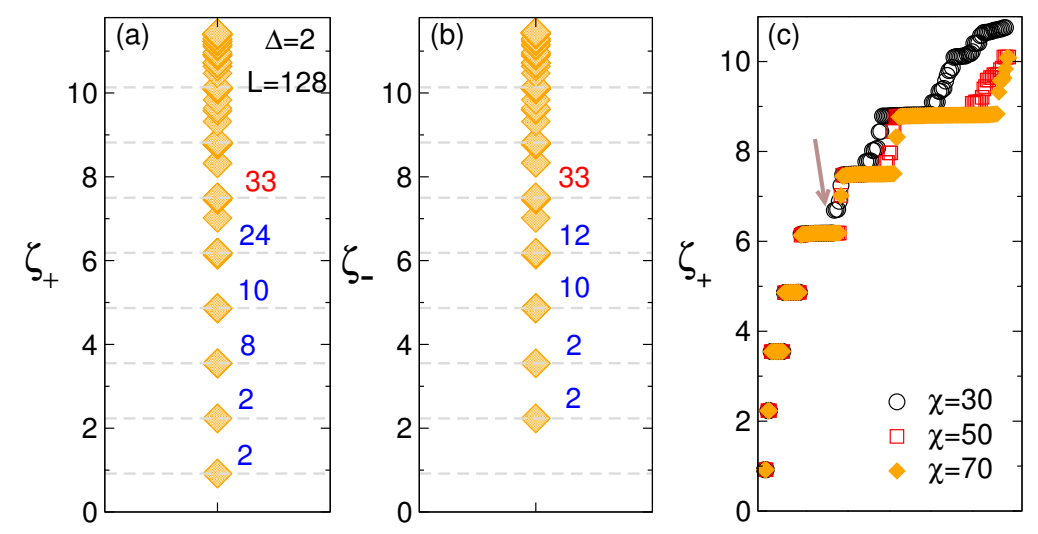

Figure 5. TEBD results for the negativity spectrum of two adjacent intervals in the gapped XXZ spin chain. The data are for a chain with $L=128$, the intervals $\ell_{1}=\ell_{2}=L / 4$, and $\Delta=2$. (a)(b) Negativity spectrum levels $\zeta_{ \pm}$in the sector of positive and negative eigenvalues of $\rho_{A}^{T_{2}}$. The accompanying numbers are the level degeneracies. The horizontal dashed lines are the analytical results for large intervals. (c) $\zeta_{+}$at $\Delta=2$ and $L=128$, obtained using different values of the bond dimension $\chi=30,50,70$. Finite $\chi$ effects are visible in the higher part of the spectrum (arrow).

of a $\chi^{2} \times \chi^{2}$ matrix. The computational cost is $\chi^{6}$. In our setup, to avoid boundary effects, the two intervals $A_{1} \cup A_{2}$ are placed at the centre of the chain. Figures 5 (a) and (b) show the positive and negative levels $\zeta_{+}$and $\zeta_{-}$of the negativity spectrum. The horizontal dash-dotted line are the expected results in the thermodynamic limit (15) and they match the numerical data perfectly in the lower part of the spectrum. The accompanying numbers are the spectrum degeneracies as obtained from TEBD. For the lower part of the negativity spectrum $(\zeta \lesssim 6)$, the degeneracy counting is in agreement with the expected result in the thermodynamic limit reported in Figure 4 . For $\zeta \gtrsim 6$ deviations are present. This is due to both the finite bond dimension of the TEBD and the finite size of the chain. Similar finite-size effects are observed in the entanglement spectrum of the XXZ chain [50]. Finite $\chi$ effects are illustrated in Figure 5 (c) focusing on the positive part of the negativity spectrum. The different symbols are TEBD results for different values of $\chi$. The negativity spectrum levels clearly converged for $\zeta \lesssim 6$, while higher levels vary upon changing $\chi$.

\section{Moments of the partial transpose and logarithmic negativity}

The knowledge of the negativity spectrum can be used to provide exact results for the moments of the partial transpose and the logarithmic negativity in the gapped XXZ chain, as we are going to do now. By definition, the logarithmic negativity of two adjacent intervals is

$\mathcal{E}_{A_{1}: A_{2}} \equiv \operatorname{Tr}\left|\rho_{A_{1} \cup A_{2}}^{T_{2}}\right|=\sum \lambda_{+}-\sum \lambda_{-}=e^{-\zeta_{0}} \sum_{k=0}^{\infty}\left[q_{+}(k)+q_{-}(k)\right] e^{-k \epsilon}$.

The sum is easily done using (14). Using also the explicit value of $\zeta_{0}(17)$, the logarithmic 


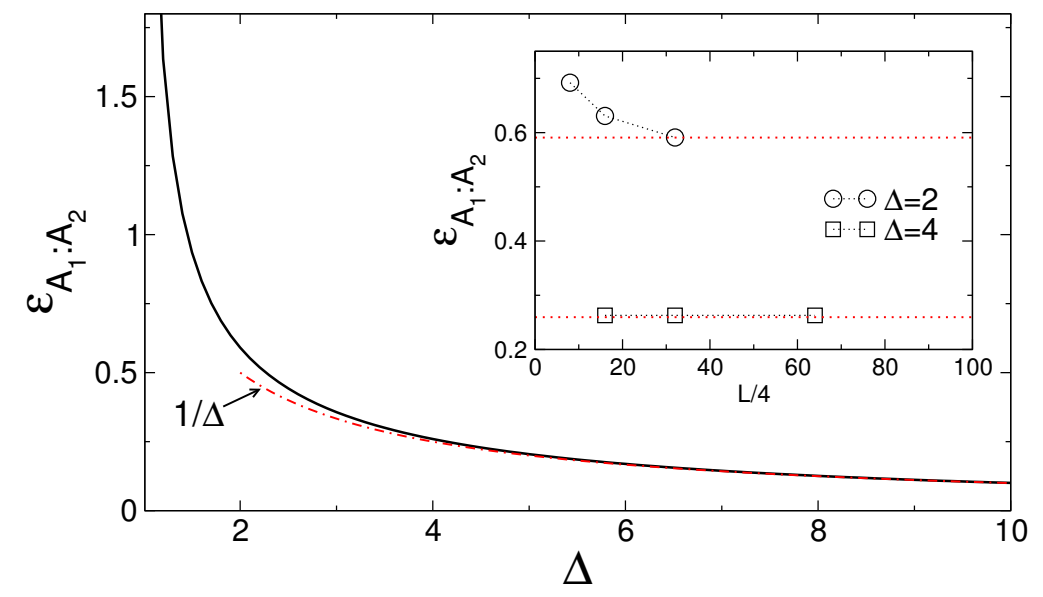

Figure 6. Exact results for the logarithmic negativity $\mathcal{E}$ of two adjacent intervals in the XXZ spin chain in the thermodynamic limit plotted as a function of the anisotropy $\Delta$. The dash-dotted line is first order expansion for $\Delta \rightarrow \infty$. Inset: Comparison with numerics for $\Delta=2$ and $\Delta=4$. The symbols are TEBD results plotted as a function of the intervals length $L / 4$ with $L$ the chain length. The dotted lines are the theoretical predictions in the thermodynamic limit.

negativity of two adjacent intervals, in the thermodynamic limit, is

$$
\mathcal{E}_{A_{1}: A_{2}}=\ln \frac{G^{2}\left(e^{-\epsilon}\right)}{G\left(e^{-2 \epsilon}\right)} .
$$

Via the relation $e^{-\epsilon}=\Delta-\sqrt{\Delta^{2}-1}$, this gives the behaviour of $\mathcal{E}_{A_{1}: A_{2}}$ as a function of the anisotropy $\Delta$ as shown in Figure 6. In the limit $\Delta \rightarrow \infty, \mathcal{E}_{A_{1}: A_{2}}$ vanishes, as a consequence of the vanishing of the bulk correlation length. The expansion for large $\Delta$ gives $\mathcal{E}_{A_{1}: A_{2}}=1 / \Delta$, which is reported as dash-dotted line in Figure 6 , and it accurately describes the behaviour of $\mathcal{E}_{A_{1}: A_{2}}$ for $\Delta \gtrsim 4$. The inset in Figure 6 shows the comparison between finite-size TEBD results for $\mathcal{E}_{A_{1}: A_{2}}$ plotted as a function of the chain size $L$, and the thermodynamic limit result (20). While finite-size corrections are present for the smaller chains, it is clear that as $L \rightarrow \infty$ the data approach (20).

We now discuss the moments of the partial transpose $\operatorname{Tr}\left(\rho_{A_{1} \cup A_{2}}^{T_{2}}\right)^{n}$. Using (14), their value in the thermodynamic limit is

$$
\ln \operatorname{Tr}\left(\rho_{A_{1} \cup A_{2}}^{T_{2}}\right)^{n}= \begin{cases}(1-n) \ln 2-3 n \ln G\left(e^{-2 \epsilon}\right)+2 \ln G\left(e^{-n \epsilon}\right) G\left(e^{-2 n \epsilon}\right) & n \text { even, } \\ (1-n) \ln 2-3 n \ln G\left(e^{-2 \epsilon}\right)+3 \ln G\left(e^{-2 n \epsilon}\right) & n \text { odd. }\end{cases}
$$

Note the dependence on the parity of $n$, alike systems described by conformal field theory [11]. By taking the analytic continuation $n \rightarrow 1$ from the result for $n$ even, the logarithmic negativity (20) is recovered. On the other hand the analytic continuation for $n$ odd gives the normalisation $\operatorname{Tr} \rho_{A_{1} \cup A_{2}}^{T_{2}}=1$, as it should. 


\section{Critical behaviour close to $\Delta=1$ and unusual scaling corrections}

We now discuss the critical behaviour of the moments of the partial transpose and of the logarithmic negativity in the vicinity of the critical point at $\Delta=1$, and we also study the scaling corrections. Unusual scaling corrections are known to affect entanglementrelated quantities [63-65]. In contrast with standard scaling corrections, their exponents depend on the replica index $n$. Moreover, while usual scaling corrections arise due to bulk irrelevant operators, the unusual ones are due to the local insertion of a relevant operator near the conical singularities $[65,66]$ introduced to calculate entanglement in the replica trick $[53,67,68]$. They are generically expected to appear also in gapped systems in the vicinity of a quantum critical point, as shown for the entanglement entropies [64] and as we are going to show here for the moments of the partial transpose.

For the gapped XXZ chain the relation between the correlation length and the anisotropy $\Delta$ is known exactly. In the limit $\Delta \rightarrow 1$ (equivalently $\epsilon \rightarrow 0$ ), i.e., close to the conformal quantum critical point, one has

$$
\ln \xi=\frac{\pi^{2}}{2 \epsilon}+\mathcal{O}\left(\epsilon^{0}\right)
$$

The strategy to derive the critical behaviour of the moments of the partial transpose is to consider the limit $\epsilon \rightarrow 0$, expanding (21) in powers of $\xi$. Similar to the moments of the reduced density matrix [64], the relations in (21) are not suitable for taking the limit $\epsilon \rightarrow 0$. It is convenient to rewrite (21) using the Poisson summation formula

$$
\sum_{j=-\infty}^{\infty} g(|\epsilon j|)=\frac{2}{\epsilon} \sum_{k=-\infty}^{\infty} \hat{g}\left(\frac{2 \pi k}{\epsilon}\right)
$$

where $\hat{g}(y)$ is the cosine transform of $g(x)$, defined as

$$
\hat{g}(y) \equiv \int_{0}^{\infty} g(x) \cos (y x) d x .
$$

Thus, defining

$$
h_{n}(x) \equiv \ln \left(1+e^{-n x}\right), \quad \text { with } \quad \hat{h}_{n}(y)=\frac{n}{2 y^{2}}-\frac{\pi}{2 y} \operatorname{csch} \frac{\pi y}{n},
$$

one has

$$
\ln G\left(z^{n}\right)=\frac{1}{2} \sum_{j=-\infty}^{\infty} h_{n}(|j \epsilon|)-\frac{\ln 2}{2}=\sum_{k=-\infty}^{\infty}\left[\frac{n \epsilon}{8 \pi^{2} k^{2}}-\frac{1}{4 k} \operatorname{csch} \frac{2 \pi^{2} k}{n \epsilon}\right]-\frac{\ln 2}{2},
$$

where in the last step we used (23). Plugging (26) in (21), one obtains for odd $n_{o}$

$\ln \operatorname{Tr}\left(\rho_{A}^{T_{2}}\right)^{n_{o}}=-\frac{\pi^{2}}{8 \epsilon}\left(n_{o}-\frac{1}{n_{o}}\right)+\frac{1}{2}\left(n_{o}-1\right) \ln 2+3 \sum_{k=1}^{\infty}\left[\frac{n_{o}}{2 k} \operatorname{csch} \frac{\pi^{2} k}{\epsilon}-\frac{1}{2 k} \operatorname{csch} \frac{\pi^{2} k}{n_{o} \epsilon}\right]$,

while for $n_{e}$ even one has

$$
\begin{aligned}
\ln \operatorname{Tr}\left(\rho_{A}^{T_{2}}\right)^{n_{e}}=- & \frac{\pi^{2}}{8 \epsilon}\left(n_{e}-\frac{2}{n_{e}}\right)+\frac{1}{2}\left(n_{e}-2\right) \ln 2 \\
& +\sum_{k=1}^{\infty}\left[\frac{3 n_{e}}{2 k} \operatorname{csch} \frac{\pi^{2} k}{\epsilon}-\frac{1}{k} \operatorname{csch} \frac{\pi^{2} k}{n_{e} \epsilon}-\frac{1}{k} \operatorname{csch} \frac{2 \pi^{2} k}{n_{e} \epsilon}\right] .
\end{aligned}
$$


In (27) and (28) the terms $\propto 1 / \epsilon$ correspond to $k=0$. From (27) and (28), using (22), one obtains that $\ln \operatorname{Tr}\left(\rho_{A}^{T_{2}}\right)^{n}$ diverges in the limit $\epsilon \rightarrow 0$ as

$$
\ln \operatorname{Tr}\left(\rho_{A}^{T_{2}}\right)^{n} \simeq \begin{cases}-\frac{1}{4}\left(n_{o}-\frac{1}{n_{o}}\right) \ln \xi+O\left(\xi^{0}\right) & n_{o} \text { odd } \\ -\frac{1}{4}\left(n_{e}-\frac{2}{n_{e}}\right) \ln \xi+O\left(\xi^{0}\right) & n_{e} \text { even }\end{cases}
$$

Notice that these results are equivalent to the CFT scaling $[11,12]$ after the replacing $\ell_{1,2} \rightarrow \xi$, as one would naively expect from a simple scaling reasoning. Taking the limit $n_{e / o} \rightarrow \infty$ one gets the scaling of the largest eigenvalue of $\operatorname{Tr}\left(\rho_{A}^{T_{2}}\right)^{n}$

$$
\ln \lambda_{0}=-\frac{\pi^{2}}{8 \epsilon}+\frac{\ln 2}{2}+O(\epsilon)
$$

which agrees with the expansion of (18) close to $\epsilon=0$. The scaling of the logarithmic negativity is instead

$$
\mathcal{E}_{A_{1}: A_{2}}=\lim _{n_{e} \rightarrow 1} \ln \operatorname{Tr}\left(\rho_{A}^{T_{2}}\right)^{n_{e}}=\frac{\pi^{2}}{8 \epsilon}-\frac{\ln 2}{2}+O(\epsilon),
$$

which agrees with the expansions of (20).

Finally, the sums in (27) and (28) give rise to scaling corrections in the limit $\epsilon \rightarrow 0$. Using the asymptotic behaviour $\operatorname{csch}(x) \propto e^{-x}$ for $x \rightarrow+\infty$, one obtains corrections of the form $\xi^{-2 k}$ from the first term in the square brackets in (27) and (28). The last

two terms in (27) and (28) give corrections of the form $\xi^{2 k / n}$ and $\xi^{4 k / n}$ which are the "expected" unusual corrections, i.e., they have the same exponents appearing in the corrections for the moments of the reduced density matrix [64].

\section{Negativity spectrum distribution}

In this section we investigate the distribution of the negativity spectrum which is defined as

$$
P(\lambda) \equiv \sum_{i} \delta\left(\lambda-\lambda_{i}\right)
$$

as sum over the eigenvalues $\lambda_{i}$ of the partial transpose. So far, this has been investigated only for conformal field theories [20]. Using the explicit expressions of the eigenvalues of the partial transpose and their degeneracy, in the XXZ spin chain $P(\lambda)$ can be written as

$$
P(\lambda)=\sum_{k=0}^{\infty} \delta\left(\lambda-\lambda_{0} e^{-k \epsilon}\right) q_{+}(k)+\sum_{k=0}^{\infty} \delta\left(\lambda-\lambda_{0} e^{-k \epsilon}\right) q_{-}(k),
$$

with $\lambda_{0}$ being the largest eigenvalue of the partial transpose (18). In the limit of large $k$ (i.e. for small $\lambda$ of the partial transpose) (32) approaches a continuous distribution which can be determined from the asymptotic behaviour of the degeneracy $q_{ \pm}(k)$ for large $k$. In Appendix A we use the Meinardus theorem to obtain the large $k$ behaviours

$$
q_{ \pm}(k) \simeq\left\{\begin{array}{cc}
\frac{k^{-\frac{3}{4}}}{8 \sqrt{2}}\left(e^{\pi \sqrt{k}} \pm 2^{\frac{5}{4}} e^{\frac{\pi}{\sqrt{2}} \sqrt{k}}\right) & \text { even } k \\
\frac{k^{-\frac{3}{4}}}{8 \sqrt{2}} e^{\pi \sqrt{k}} & \text { odd } k
\end{array}\right.
$$


Consequently one can write

$P\left(\lambda_{k}\right) \frac{d \lambda_{k}}{d k}=\theta\left(\lambda_{k}\right) \frac{k^{-\frac{3}{4}}}{8 \sqrt{2}}\left(e^{\pi \sqrt{k}}+2^{\frac{1}{4}} e^{\frac{\pi}{\sqrt{2}} \sqrt{k}}\right)+\theta\left(-\lambda_{k}\right) \frac{k^{-\frac{3}{4}}}{8 \sqrt{2}}\left(e^{\pi \sqrt{k}}-2^{\frac{1}{4}} e^{\frac{\pi}{\sqrt{2}} \sqrt{k}}\right)$,

where $\lambda_{k}=\lambda_{0} e^{-k \epsilon}$ is the $k$-th eigenvalue. The approximation of the sum in (32) with an integral requires that $\ln \left(\lambda_{0} /\left|\lambda_{k}\right|\right) \gg \epsilon$. Since $\epsilon=\operatorname{arccosh} \Delta$, this implies that the larger $\Delta$ is, the smaller $\left|\lambda_{k}\right|$ has to be for (35) to be valid.

To make contact with Ref. [20], it is worth considering the number distribution function $n(\lambda)$, which for $\lambda>0(\lambda<0)$ counts the number of eigenvalues of the partial transpose larger (smaller) than $\lambda$. It is useful to treat the cases $\lambda>0$ and $\lambda<0$ separately, defining $n_{ \pm}(|\lambda|)$ as

$$
n_{ \pm}(|\lambda|)=\sum_{k=0}^{\infty} \theta\left(|\lambda|-\lambda_{0} e^{-k \epsilon}\right) q_{ \pm}(k)=\sum_{k=0}^{\infty} \theta\left(\ln \left(\lambda_{0} /|\lambda|\right)-k \epsilon\right) q_{ \pm}(k) .
$$

The asymptotic behaviour of $n_{ \pm}(|\lambda|)$ is straightforwardly obtained from that of $P(\lambda)$, and it is conveniently written in the suggestive form

$$
n_{ \pm}(\lambda)=\frac{1}{\sqrt{2 \eta} \pi}\left(\frac{1}{4} e^{\pi \eta} \pm \frac{1}{2^{\frac{5}{4}}} e^{\frac{\pi}{\sqrt{2}} \eta}\right), \quad \text { with } \eta \equiv \sqrt{\frac{\ln \left(\lambda_{0} /|\lambda|\right)}{\epsilon}},
$$

where all the dependence on $\lambda$ is encoded in the universal scaling variable $\eta$, without any explicit dependence on the parameters of the model. This is very similar to what found in conformal field theories [20], but a more quantitative comparison between the two results requires a careful analysis (with consequences also on the entanglement spectrum) which will be discussed elsewhere [69].

In the limit $\eta \rightarrow \infty$ both $n_{+}(\lambda)$ and $n_{-}(\lambda)$ exhibit the same asymptotic behaviour as

$$
n_{ \pm}(\lambda) \propto \frac{1}{4 \pi \sqrt{2 \eta}} \exp (\pi \eta)
$$

which means that the number distribution does not depend on the sign of the eigenvalues of the partial transpose. Similar to (38), for the negativity spectrum of conformal field theories [46] the distribution of the small eigenvalues of the partial transpose is exponential, and it does not depend on the sign of the eigenvalues. The same asymptotic behaviour for $|\lambda| \rightarrow 0$ is already visible for $\eta \equiv\left(\ln \left(\lambda_{0} /|\lambda|\right) / \epsilon\right)^{\frac{1}{2}} \approx 4$, see Figure 7 .

We now provide numerical evidence supporting the analytical result for the number distribution $n_{ \pm}(\lambda)$ in the gapped XXZ chain. It is not instructive to compare (37) with the TEBD results since we already showed that these agree well with the CTM prediction in the thermodynamic limit and so we prefer to contrast the exact CTM result to the asymptotic prediction. This comparison is illustrated in Figure 7, plotting $n_{+}(\lambda)$ and $n_{-}(\lambda)$ versus the scaling variable $\eta=\left(\ln \left(\lambda_{0} /|\lambda|\right) / \epsilon\right)^{\frac{1}{2}}$, which is a plot independent from $\Delta$. The dash-dotted lines are the analytical results (37), and are in excellent agreement with the numerics already for $\eta \gtrsim 2$. 


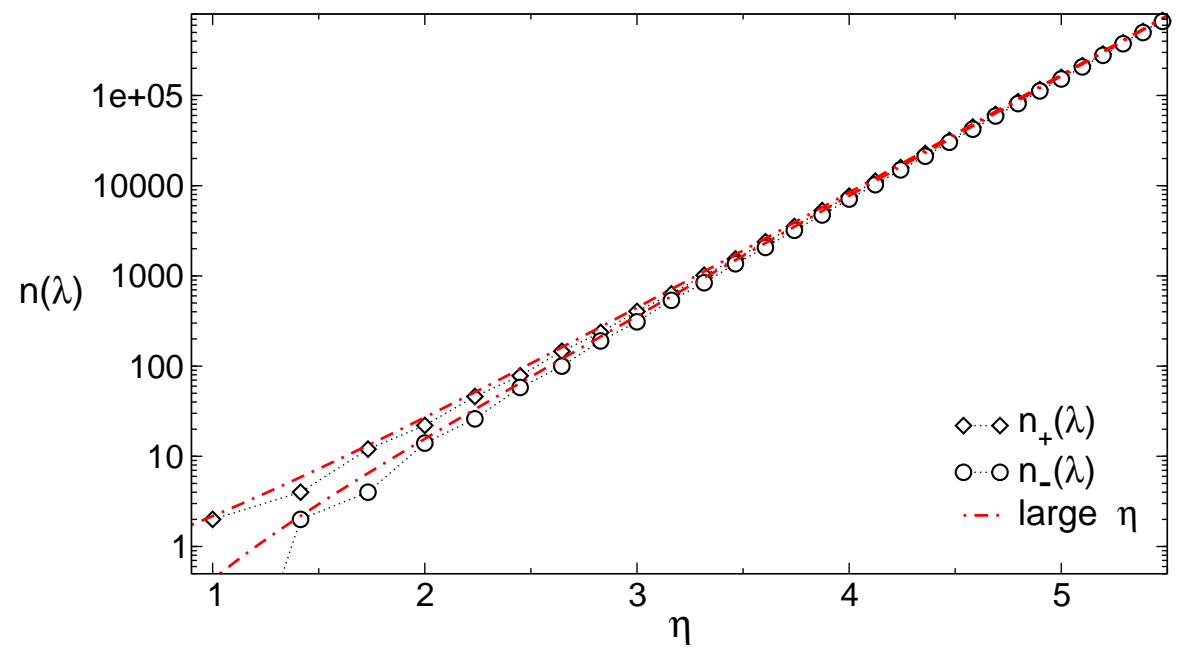

Figure 7. Negativity spectrum of two adjacent intervals in the XXZ chain: number distribution $n(\lambda)$ plotted as a function of $\eta=\left(\left(\ln \left(\lambda_{0} /|\lambda|\right) / \epsilon\right)^{\frac{1}{2}}\right.$, which does not dependent on $\Delta$. The symbols correspond to the exact result in the thermodynamic limit given by (36). Diamonds (circles) correspond to positive (negative) eigenvalues. The continuous lines are the asymptotic behaviours for large $\eta$ (37) which describe well the data for $\eta \gtrsim 2$.

\section{Conclusions}

We investigated the negativity spectrum of two adjacent intervals in generic gapped one-dimensional systems. We demonstrated that in the limit of large intervals the negativity spectrum can be reconstructed completely from the entanglement spectrum of the bipartite system. Our main result is formula (8). The result relies upon two main physical ingredients. First, the contributions to the negativity spectrum originating from different boundaries (namely, the boundary between the two subsystems and the subsystems and the rest) are factorized. Second, due to the finite correlation length, around the boundary between the two intervals the system is described effectively by a pure state.

As an application of our result we discussed in detail the negativity spectrum of the spin-

$\frac{1}{2}$ Heisenberg XXZ chain in the gapped phase for $\Delta>1$. We considered separately the negative and positive eigenvalues of the partial transpose. In both cases the negativity spectrum levels are equally spaced, similar to the entanglement spectrum. The level spacing, however, is half that of the entanglement spectrum. Negativity spectrum levels are highly degenerate. The degeneracies can be characterised analytically in terms of the partition of the integers. This allowed us to provide exact results for the logarithmic negativity, the moments of the partial transpose, and the associated unusual scaling corrections. Finally, we derived analytically the distribution of the negativity spectrum levels. We found that the number distribution is independent of $\Delta$. Moreover, for the small eigenvalues of the partial transpose it does not depend on the eigenvalue sign, as for the negativity spectrum of conformal field theories [46]. We provided accurate 
numerical checks of our results using TEBD simulations.

We finish by discussing some directions for future work. First, we considered the negativity spectrum of the gapped XXZ chain, which is an integrable model (but our results are valid for an arbitrary model solvable by corner transfer matrix as e.g. those in Refs. 56-60). It would be interesting to generalise our results for the distribution of the entanglement spectrum levels (for instance (37)) to non-integrable systems. Moreover, the negativity spectrum distribution could have applications also to disordered models exhibiting many body localisation, generalising the results for the entanglement spectrum [70]. Finally, it would also interesting to clarify the signatures of topological order in the negativity spectrum.

\section{Acknowledgments}

V. A. acknowledges support from the European Union's Horizon 2020 research and innovation programme under the Marie Sklodowoska-Curie grant agreement No 702612 $O E M B S$.

\section{Appendix A. Meinardus theorem}

Here we review the main result of the Meinardus theorem (see Ref. 71 for more details). The power of Meinardus theorem is that it allows to obtain the asymptotic behaviour of a large class of restricted integer partition $r(k)$ for large $k$. Specifically, let us consider a generic $r(k)$ that is defined via the generating function $f(q)$ as

$$
f(q) \equiv 1+\sum_{k=1}^{\infty} r(k) q^{k}=\prod_{k=1}^{\infty}\left(1-q^{k}\right)^{-\alpha_{k}}
$$

where $|q|<1$ and $\alpha_{k}$ are non-negative numbers. Different types of restricted partitions can be obtained by choosing different sequences $\alpha_{k}$. It is convenient for the following to define an auxiliary Dirichlet series $D(s)$ as

$$
D(s) \equiv \sum_{n=1}^{\infty} \frac{\alpha_{n}}{n^{s}}
$$

The Meinardus theorem states that the leading behaviour of $r(k)$ for large $k$ is given as

$$
r(k) \propto C n^{\kappa} \exp \left\{n^{\alpha /(\alpha+1)}\left(1+\frac{1}{\alpha}\right)[A \Gamma(\alpha+1) \zeta(\alpha+1)]^{1 /(\alpha+1)}\right\} .
$$

Here

$$
C \equiv e^{D^{\prime}(0)}[2 \pi(1+\alpha)]^{-\frac{1}{2}}[A \Gamma(\alpha+1) \zeta(\alpha+1)]^{(1-2 D(0)) /(2+2 \alpha)},
$$

$\kappa$ is given as

$$
\kappa=\frac{D(0)-1-\alpha / 2}{1+\alpha}
$$

and $A$ is the residue of the first order pole of $D(s)($ cf. (A.2)) at $s=\alpha$. 
We now proceed to apply the Meinardus theorem to the large $k$ behaviour of $q_{ \pm}(k)$ of our interest. To proceed, it is convenient to first derive the asymptotic behaviour of the combinations $q_{+} \pm q_{-}$. From (14), one has that the generating function of $\left(q_{+}+q_{-}\right) / 2$ is $G^{2}\left(x^{2}\right) G^{2}(x)$,

$$
G^{2}\left(x^{2}\right) G^{2}(x)=\prod_{k=1}^{\infty}\left(1+x^{2 k}\right)^{2}\left(1+x^{k}\right)^{2}=\prod_{k=1}^{\infty}\left(1-x^{k}\right)^{\alpha_{k}^{\prime}},
$$

where $\alpha_{k}^{\prime}=2$ for $k$ not divisible by four, and zero otherwise. Using (A.2), one has

$$
D(s)=2^{1-2 s}\left(2^{2 s}-1\right) \zeta(s) .
$$

$D(s)$ has a pole of order one at $s=1$ and residue $A=3 / 2$, implying $\alpha=1$ in (A.3). Using the Meinardus theorem, one obtains

$$
q_{+}+q_{-} \propto \frac{1}{4 \sqrt{2}} k^{-\frac{3}{4}} e^{\pi \sqrt{k}}, \quad k \rightarrow \infty .
$$

On the other hand, one has that the generating function for $\left(q_{+}-q_{-}\right) / 2$ is

$$
G^{3}\left(x^{2}\right)=\prod_{k=1}^{\infty}\left(1-x^{k}\right)^{\alpha_{k}^{\prime \prime}}
$$

where $\alpha_{k}^{\prime \prime}=3$ if $k \bmod 4=2$, and zero otherwise. It is straightforward to check that in this case

$$
D(s)=\frac{3}{2^{2 s}}\left(2^{s}-1\right) \zeta(s) .
$$

This implies that $\alpha=1$ and $A=3 / 4$ in (A.3). The Meinardus theorem now yields

$$
q_{+}-q_{-} \propto\left\{\begin{array}{cc}
2^{-\frac{5}{4}} k^{-\frac{3}{4}} e^{\frac{\pi}{\sqrt{2}} \sqrt{k}} & k \text { even } \\
0 & k \text { odd } .
\end{array}\right.
$$

Using (A.8) and (A.11), one obtains for large $k$

$$
q_{ \pm}(k) \simeq\left\{\begin{array}{cc}
\frac{k^{-\frac{3}{4}}}{8 \sqrt{2}}\left(e^{\pi \sqrt{k}} \pm 2^{\frac{5}{4}} e^{\frac{\pi}{\sqrt{2}} \sqrt{k}}\right) & \text { even } k \\
\frac{k^{-\frac{3}{4}}}{8 \sqrt{2}} e^{\pi \sqrt{k}} & \text { odd } k
\end{array}\right.
$$

which is the result reported in the main text (cf. (34)).

\section{References}

[1] L. Amico, R. Fazio, A. Osterloh, and V. Vedral, Rev. Mod. Phys. 80, 517 (2008).

[2] P. Calabrese, J. Cardy, and B. Doyon Eds, J. Phys. A 42, 500301 (2009).

[3] J. Eisert, M. Cramer, and M. B. Plenio, Rev. Mod. Phys. 82, 277 (2010).

[4] N. Laflorencie, Phys. Rep. 643, 1 (2016).

[5] A. Peres, Phys. Rev. Lett. 77, 1413 (1996).

[6] K Zyczkowski, P. Horodecki, A. Sanpera, and M. Lewenstein, Phys. Rev. A 58, 883 (1998).

[7] K. Zyczkowski, Phys. Rev. A 60, 3496 (1999).

[8] J. Lee, M. S. Kim, Y. J. Park, and S. Lee, J. Mod. Opt. 47, 2151 (2000);

J. Eisert and M. B. Plenio, J. Mod. Opt. 46, 145 (1999).

[9] G. Vidal and R. F. Werner, Phys. Rev. A 65, 032314 (2002). 
[10] M. B. Plenio, Phys. Rev. Lett. 95, 090503 (2005);

J. Eisert, quant-ph/0610253.

[11] P. Calabrese, J. Cardy, and E. Tonni, Phys. Rev. Lett. 109, 130502 (2012).

[12] P. Calabrese, J. Cardy, and E. Tonni, J. Stat. Mech. P02008 (2013).

[13] P. Calabrese, L. Tagliacozzo, and E. Tonni, J. Stat. Mech. (2013) P05002.

[14] S. Furukawa, V. Pasquier, and J. Shiraishi, Phys. Rev. Lett. 102, 170602 (2009);

P. Calabrese, J. Cardy, and E. Tonni, J. Stat. Mech. P11001 (2009);

P. Calabrese, J. Cardy, and E. Tonni, J. Stat. Mech. P01021 (2011);

V. Alba, L. Tagliacozzo, and P. Calabrese, Phys. Rev. B 81, 060411 (2010);

M. Fagotti and P. Calabrese, J. Stat. Mech. (2010) P04016;

V. Alba, L. Tagliacozzo, and P. Calabrese, J. Stat. Mech. P06012 (2011);

M. A. Rajabpour and F. Gliozzi, J. Stat. Mech. (2012) P02016;

A. Coser, L. Tagliacozzo, and E. Tonni, J. Stat. Mech. (2014) P01008.

[15] H. Wichterich, J. Molina-Vilaplana, and S. Bose, Phys. Rev. A 80, 010304 (2009).

[16] S. Marcovitch, A. Retzker, M. B. Plenio and B. Reznik, Phys. Rev. A 80, 012325 (2009).

[17] H. Wichterich, J. Vidal, and S. Bose, Phys. Rev. A 81, 032311 (2010).

[18] P. Calabrese, J. Cardy, and E. Tonni, J. Phys. A 48, 015006 (2015).

[19] M. Kulaxizi, A. Parnachev, and G. Policastro, JHEP 09 (2014) 010.

[20] P. Ruggiero, V. Alba, and P. Calabrese, Phys. Rev. B 94, 035152 (2016).

[21] A. Coser, E. Tonni and P. Calabrese, J. Stat. Mech. P12017 (2014).

[22] M. Hoogeveen and B. Doyon, Nucl. Phys. B 898, 78 (2015).

[23] V. Eisler and Z. Zimboras, New J. Phys. 16, 123020 (2014).

[24] X. Wen, P.-Y. Chang, and S. Ryu, Phys. Rev. B 92, 075109 (2015).

[25] M. Rangamani, and M. Rota, JHEP 10 (2014) 060;

E. Perlmutter, M. Rangamani, and M. Rota, Phys. Rev. Lett. 115, 171601 (2015).

[26] O. Blondeau-Fournier, O. Castro-Alvaredo, and B. Doyon, J. Phys. A 49, 125401 (2016).

[27] D. Bianchini and O. A. Castro-Alvaredo, Nucl. Phys. B 913, 879 (2016).

[28] Y. A. Lee and G. Vidal, Phys. Rev. A 88, 042318 (2013).

[29] C. Castelnovo, Phys. Rev. A 88, 042319 (2013);] C. Castelnovo, Phys. Rev. A 89, 042333 (2014).

[30] A. Bayat, S. Bose, P. Sodano, and H. Johannesson, Phys. Rev. Lett. 109, 066403 (2012);

A. Bayat, P. Sodano, and S. Bose, Phys. Rev. B 81, 064429 (2010).

[31] A. Bayat, H. Johannesson, S. Bose, and P. Sodano, Nat. Comm. 5, 3784 (2014).

[32] B. Alkurtass, A. Bayat, I. Affleck, S. Bose, H. Johannesson, P. Sodano, E. S. Sorensen, and K. Le Hur, Phys. Rev. B 93, 081106 (2016).

[33] X. Wen, S. Matsura, and S. Ryu, Phys. Rev. B 93, 245140 (2016).

[34] X. Wen, P.-Y. Chang, and S. Ryu, JHEP 09 (2016) 012.

[35] K. Audenaert, J. Eisert, M. B. Plenio, and R. F. Werner, Phys. Rev. A 66, 042327 (2002).

[36] V. Eisler and Z. Zimboras, Phys. Rev. B 93, 115148 (2016).

[37] C. De Nobili, A. Coser, and E. Tonni, J. Stat. Mech. (2016) 083102.

[38] V. Eisler and Z. Zimboras, New J. Phys. 17053048 (2015).

[39] A. Coser, E. Tonni, and P. Calabrese, J. Stat. Mech. (2015) P08005.

[40] A. Coser, E. Tonni, and P. Calabrese, J. Stat. Mech. (2016) 033116;

A. Coser, E. Tonni, and P. Calabrese, J. Stat. Mech. (2016) 053109.

[41] P.-Y. Chang and X. Wen, Phys. Rev. B 93, 195140 (2016).

[42] C. P. Herzog and Y. Wang, J. Stat. Mech. (2016) 073102.

[43] H. Shapourian, K. Shiozaki, and S. Ryu, arXiv:1607.03896.

[44] H. Shapourian, K. Shiozaki, and S. Ryu, arXiv:1611.07536.

[45] J. Eisert, V. Eisler, Z. Zimboras, arXiv:1611.08007.

[46] P. Ruggiero, V. Alba, and P. Calabrese, Phys. Rev. B 94, 195121 (2016).

[47] H. A. Carteret, Phys. Rev. Lett. 94, 040502 (2005)

[48] P. Calabrese and A. Lefevre, Phys. Rev A 78, 032329 (2008). 
[49] K. Okunishi, Y. Hieida, and Y. Akutsu, Phys. Rev. E 59, R6227 (1999).

[50] V. Alba, M. Haque, and A. M. Läuchli, Phys. Rev. Lett. 108, 227201 (2012).

[51] T. Nishino, J. Phys. Soc. Jpn. 74, 3598 (1995);

T. Nishino and K. Okunishi, Lect. Notes Phys. 478, 167 (1997).

[52] I. Peschel, M. Kaulke, and O. Legeza, Ann. Physik (Leipzig) 8, 153 (1999).

[53] P. Calabrese and J. Cardy, J. Stat. Mech. P06002 (2004).

[54] V. Eisler and I. Peschel, J. Phys. A 42, 504003 (2009).

[55] R. J. Baxter, Exactly Solved Models in Statistical Mechanics, 1982 Academic Press, San Diego.

[56] R. Weston, J. Stat. Mech. L03002 (2006).

[57] E. Ercolessi, S. Evangelisti, and F. Ravanini, Phys. Lett. A 374, 2101 (2010).

[58] E. Ercolessi, S. Evangelisti, F. Franchini, and F. Ravanini, Phys. Rev. B 83, 012402 (2011);

E. Ercolessi, S. Evangelisti, F. Franchini, and F. Ravanini, Phys. Rev. B 85, 115428 (2012);

E. Ercolessi, S. Evangelisti, F. Franchini, and F. Ravanini, Phys. Rev. B 88, 104418 (2013).

[59] A. De Luca and F. Franchini, Phys. Rev. B 87, 045118 (2013).

[60] D. Bianchini and F. Ravanini, J. Phys. A 49 (2016) 154005.

[61] G. Vidal, Phys. Rev. Lett. 91, 147902 (2003).

[62] G. Vidal, Phys. Rev. Lett. 93, 040502 (2004).

[63] P. Calabrese, M. Campostrini, F. Essler, and B. Nienhuis, Phys. Rev. Lett. 104095701 (2010);

P. Calabrese and F. H. L. Essler, J. Stat. Mech. (2010) P08029;

M. Fagotti and P. Calabrese, J. Stat. Mech. P01017 (2011).

[64] P. Calabrese, J. Cardy, and I. Peschel, J. Stat. Mech. P09003 (2010).

[65] J. Cardy and P. Calabrese, J. Stat. Mech. (2010) P04023.

[66] K Ohmori and Y. Tachikawa, J. Stat. Mech. P04010 (2015).

[67] C. G. Callan and F. Wilczek, Phys. Lett. B 333, 55 (1994).

[68] C. Holzhey, F. Larsen and F. Wilczek, Nucl. Phys. B 424, 443 (1994).

[69] V. Alba, P. Calabrese, and E. Tonni, in preparation.

[70] M. Serbyn, A. A. Michailidis, D. A. Abanin, and Z. Papic, Phys. Rev. Lett. 117, 160601 (2016).

[71] G. E. Andrews, The theory of partitions, Cambridge University Press, Cambridge (1984). 